\title{
Bewerbungsverfahren um ein Clinical Fellowship in den USA unmittelbar nach der Facharztweiterbildung
}

Facharztausbildung in Deutschland beendet, Facharztprüfung bestanden - „what next"? „Fellowships" sind Möglichkeiten, sich in der Augenheilkunde zu spezialisieren, v. a. außerhalb Deutschlands. Ich persönlich habe mich für die USA entschieden aufgrund der exzellenten Weiterbildungsmöglichkeiten an weltweit renommierten Universitäten und weil die Kongresse der „American Academy of Ophthalmology (AAO)“ mich jedes Jahr überzeugt haben, diesen Schritt zu gehen.

\section{Clinical vs. Research}

„Fellowships" sind Trainingsprogramme mit einer Dauer von bis zu 24 Monaten nach Beendigung der Facharztausbildung zur Spezialisierung in verschiedenen Bereichen der Augenheilkunde. Es werden Weiterbildungsstellen in "cornea and anterior segment, glaucoma, medical retina, vitreoretinal surgery, uveitis, ocular oncology, oculoplastics, pathology, pediatric ophthalmology and strabismus" und "neuroophthalmology" angeboten. Eine operative Vorerfahrung sollte vorhanden sein. Unterschieden wird zwischen "Clinical" und „Research" Fellowships. Clinical Fellowships erlauben täglichen Patientenkontakt und setzen daher das Erlangen einer temporären Arztlizenz in den USA voraus - Research Fellowships können dagegen über Stipendien organisiert werden (z. B. über die Deutsche Forschungsgemeinschaft) und benötigen in der Regel keine Arztlizenz. Generell sind die Chancen, bei weniger operativen Bereichen aufgenommen zu werden, etwas höher, jedoch nicht garantiert.

\section{Voraussetzungen zur Bewerbung}

Sich für ein Clinical Fellowship in den USA zu qualifizieren ist zeitaufwendig und kostspielig, daher sollte eine entsprechende Planung frühzeitig erfolgen. Alle 3 Teile des Staatsexamens in den USA - United States Medical License Examinations (USMLEs)- müssen bestanden werden. Die Kosten pro Prüfung variieren zwischen 900 und 1000 USD. Die notwendige Vorbereitungszeit ist abhängig vom Absolventen. Ich selbst hatte mich während meines Medizinstudiums dazu entschlossen, für die Prüfungen zu lernen, und habe einige nach dem Studium absolviert. USMLE Step 3 habe ich während meines 3 . Weiterbildungsjahres in der Augenheilkunde beendet.

Das richtige Fellowship-Programm aussuchen: Eine ausgiebige Recherche, welche Universitäten bereit sind, internationale Fachärzte - sog. „International Medical Graduates (IMGs)" - aufzunehmen, ist notwendig. Hier sollte man am besten die Homepages der jeweiligen Universitäten selbstständig überprüfen und die Ausbildung/den Werdegang der aktuellen „Fellows" genauer beobachten.

Arztlizenz in den USA: Um eine Arztlizenz jeglicher Form zu erwerben, müssen die USMLEs bestanden werden. Für eine permanente Arztlizenz wird die Absolvierung einer "Accreditation Council for Graduate Medical Education(ACGME)"-akkreditierten Ausbildung verlangt. Abhängig vom Bundesstaat müssen 2-3 Jahre in einem ACGME-akkreditierten Ausbildungsprogramm absolviert werden. In der Augenheilkunde ist nur die sog. „Residency" in den USA - also die Facharztausbildung - durch die ACGME akkreditiert.

Alternativ sind verschiedene Formen von temporären Arztlizenzen vorhanden, welche abhängig von unterschiedlichen Richtlinien im jeweiligen Bundesstaat erteilt werden und die ärztliche Tätigkeit für die Dauer des Fellowships in den USA ermöglichen können.

Einige wichtige Qualifikationen für eine permanente Arztlizenz in den USA:

- United States Medical License Exami-

nations

- USMLE Step 1: MCQ

- USMLE 2 Clinical Knowledge (CK): MCQ

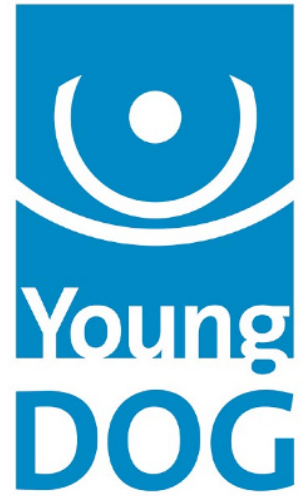

Die AG Young DOG richtet sich an junge Assistenzärzte und Nachwuchswissenschaftler. Sie fokussiert ihre Aktivitäten auf die Förderung des wissenschaftlichen Nachwuchses in der Ophthalmologie und die Vereinbarkeit von Klinik, Forschung und Familie.

Die Mitgliedschaft in der AG ist den Mitgliedern der DOG vorbehalten. Mitglied werden können Assistenzärzte, habilitierte Ärzte und Wissenschaftler sowie Professoren bis W2.

Nähere Information zur AG Young DOG finden Sie unter http://www.dog.org/?cat=137.

\section{Gabrielle N. Turski}

Medical Retina Fellow

Duke Eye Center, Duke University,

Durham, USA

\section{Korrespondenzadresse}

Gabrielle N. Turski, MD, FEBO

Medical Retina Fellow

Duke Eye Center, Duke University

Durham, NC 27705, USA

Gabrielle.Turski@duke.edu

Gabrielle.Turski@ukbonn.de

Ophthalmologe 2021 · 118:965-966 https://doi.org/10.1007/s00347-021-01464-8 ( $)$ Springer Medizin Verlag GmbH, ein Teil von Springer Nature 2021 
- USMLE 2 Clinical Skills (CS): Praktische Prüfung in den USA (aufgrund von COVID-19 findet diese Prüfung nicht mehr statt)

- USMLE Step 3: MCQ an 2 Tagen

- Educational Commission for Foreign Medical Graduates (ECFMG) Certification:

Ausstellung eines Zertifikats nachdem USMLE 1, 2CK, (2 CS) bestanden wurden

- Zwei bis 3 Jahre ACGME-akkreditiertes Training in den USA oder Kanada

\section{Observerships in den USA und "reference letters"}

Observerships - auch bekannt als „shadowing" - ermöglichen es, bereits einige Kontakte zu knüpfen, elektronische Krankenaktensysteme kennenzulernen und Erfahrungen in den USA zu sammeln. Die Absolvierung eines Observerships in den USA ist empfehlenswert, jedoch für die Bewerbung nicht zwingend notwendig. Hierfür gibt es die Möglichkeit, sich direkt an den Universitäten zu bewerben. Welche Qualifikationen notwendig sind, werden meistens auf den Homepages der Universitäten beschrieben, sonst einfach per E-Mail nachfragen, wenn Interesse besteht. Je länger das Observership, umso besser, denn zusätzlich sind "reference letters" für die Bewerbung notwendig.,"Reference letters" aus dem Heimatland sind zwar ebenfalls ausreichend, "reference letters" aus den USA werden jedoch positiv berücksichtigt. Bewerber(innen) benötigen 3, manchmal auch bis zu 4 „reference letters“, welche von Oberärzt(inn)en, mit denen man selbst einige Jahre zusammengearbeitet hat, stammen sollten und welche eine verlässliche Einschätzung über die klinischen Qualifikationen geben können. Eine der "reference letters" ist immer von dem/der Klinikleiter(in) anzufordern. Die Referenten können im Verlauf bei Fragen von den „Program-Directors" aus den USA kontaktiert werden.

\section{Bewerbung via San Francisco Matching Services: Profilerstellung}

Klinische Fellowship-Positionen werden jährlich über "San Francisco Matching Services" vergeben. Hierfür ist eine Anmel- dung bei sfmatch.org notwendig, um ein eigenes Profil zu erstellen. Dann werden die erforderlichen Informationen und Dokumente (u.a. „reference letters", CV und „personal statement ${ }^{\prime \prime}$ ) hochgeladen und die Bewerbungen direkt über das System an die ausgesuchten Fellowship-Programme versendet. Es ist wichtig zu überprüfen, welche Fellowship-Programme IMGs berücksichtigen, welche Arztlizenz notwendig ist und ob ein Visum gesponsert wird. Dann heißt es warten, bis vielleicht doch Einladungen zum Interview kommen!

\section{Interviews}

Potenzielle Kandidaten werden von dem „Program-Director“ zum Interview eingeladen. In 2020 erfolgten diese aufgrund von COVID-19 online. Die jeweilige Fakultät lernt die Kandidaten kennen und stellt ihr Fellowship-Programm vor. Einzelne Interviews mit der medizinischen Fakultät finden statt. Außerdem bekommen die Kandidaten die Möglichkeit, mit den aktuellen Fellows zu diskutieren und diese und deren Eindrücke kennenzulernen. Die individuellen Interviews können entweder mit einem einzelnen Fakultätsmitglied oder einem Komitee, bestehend aus mehreren Personen, oder mehreren verschiedenen Komitees stattfinden. Auch kann die Dauer zwischen 10 und 30 min pro separatem Interview variieren. Hier ist es sinnvoll, sich darauf vorzubereiten, die Struktur der eigenen Facharztausbildung in Deutschland und eigene klinische Erfahrungen beschreiben zu können.

\section{The Match - Rank List}

Anfang Dezember muss vom Kandidaten und den Program-Directors eine "Rank List" erstellt und im SF-Matching-System eingegeben werden. Bei der Erstellung der eigenen Rank List sollten die persönlichen Präferenzen berücksichtigt werden. Es ist auch möglich, ein Fellowship-Programm, bei welchem jemandem das Interview nicht gefallen hat, gar nicht erst zu listen. Nach abgelaufener Deadline erfolgt das Matching, sodass Mitte Dezember die Ergebnisse vorliegen. Per E-Mail wird mitgeteilt, ob eine Ausbildungsstelle erhalten wurde. Für den Fall, dass es nicht geklappt hat, besteht die Möglichkeit, sich im „Post-
Match" um Positionen, welche nicht gefüllt wurden, zu bewerben.

\section{Medical License in den USA im Überblick}

Der Prozess von Anmeldung bis zur Ausstellung einer Lizenz kann bis zu 5 Monate dauern und erfordert sehr viele Dokumente/Bescheinigungen.

Wenn keine ärztliche Ausbildung in den USA erfolgte, die Facharztausbildung aber in Deutschland abgeschlossen wurde, so ist es in einigen (jedoch nicht allen) Bundesstaaten möglich, eine „Fellowship“ oder „Faculty License“ zu beantragen. Diese ist jedoch nur an eine bestimmte Institution gebunden. Es ist daher notwendig, mit dem „Program-Director“ im Voraus zu klären, welche Lizenz erforderlich ist, um die Arbeitsstelle anzutreten. Es kann auf der Homepage der Universität zwar stehen, dass eine permanente Lizenz notwendig ist, jedoch könnte es manchmal doch vielleicht möglich sein, mit einer anderen Lizenzform akzeptiert zu werden.

Wichtig zu beachten: Absolvierung von Clinical Fellowships führt nicht direkt zur permanenten Lizenz in den USA. Die Facharztausbildung in Deutschland wird der „Residency ${ }^{\prime}$ in den USA nicht gleichgestellt und somit nicht anerkannt. Der Aufenthalt ist auf die Dauer des Fellowships begrenzt.

\section{Fazit}

Lernen, Disziplin, Geduld und entsprechende Planung erlauben es auch, die USMLEs während der Facharztausbildung zu absolvieren. Ich persönlich habe mich dazu entschlossen, mich um Fellowships in den USA zu bewerben, um mein klinisches Wissen und meine Erfahrungen auszuweiten und um meine manuellen und operativen Fertigkeiten weiterzuentwickeln und zu optimieren.

Ein Clinical Fellowship in den USA zu bekommen ist zwar kompetitiv, aber durchaus realisierbar - mehrfache Anläufe können dafür jedoch notwendig sein.

Weitere Informationen über erforderliche Qualifikationen und den gesamten Bewerbungsprozess sind auf www.ECFMG. org, www.USMLE.org, www.FSMB.org und https://sfmatch.org verfügbar.

Viel Erfolg! 